\title{
Treatment strategy for empyema with fistulas
}

\author{
Yukiyasu Takeuchi, Akio Hayashi, Yuko Kagawa, Yuya Kogita, Meinoshin Okumura
}

Department of General Thoracic Surgery, National Hospital Organization Osaka Toneyama Medical Center, 5-1-1 Toyonaka-City, Osaka, Japan Contributions: (I) Conception and design: All authors; (II) Administrative support: Y Takeuchi; (III) Provision of study materials or patients: Y Takeuchi; (IV) Collection and assembly of data: Y Takeuchi; (V) Data analysis and interpretation: Y Takeuchi; (VI) Manuscript writing: All authors; (VII) Final approval of manuscript: All authors.

Correspondence to: Yukiyasu Takeuchi, MD. Department of General Thoracic Surgery, National Hospital Organization Osaka Toneyama Medical Center, 5-1-1 Toyonaka-City, Osaka, Japan. Email: takeuchi.yukiyasu.wh@mail.hosp.go.jp.

Background: Among empyema cases, those with a fistula are considered to be intractable and often associated with a respiratory infectious disease caused by a variety of factors. In the present study, we examined the usefulness of a treatment strategy that combined endoscopic fistula closure with negative pressure wound therapy and present therapeutic outcomes of cases of empyema with fistula experienced since 2003.

Methods: We retrospectively investigated 49 cases of empyema with fistula surgically treated for the causative disease during the period from January 2003 to December 2018 by examining medical records.

Results: The causative diseases were acid-fast bacterium-related diseases such as tuberculosis, late effects of tuberculosis, or non-tuberculosis mycobacterium infection in 19, aspergillus infection in 8 , post-pneumonectomy bronchial stump fistula in 8 , post-pneumonectomy esophageal fistula in 2 , and common bacterial respiratory infection in 12 cases. The numbers of surgical procedures performed were 55 debridement or decortication, 37 open-window thoracotomy (OWT), 28 muscle flap transposition, 16 thoracoplasty, 7 omentopexy, and 3 extra-pleural pneumonectomy. The outcome was cure and discharge in $37(65.3 \%)$, discharge with OWT or a chest tube in $7(14.3 \%)$, and death in the hospital in $10(20.4 \%)$ cases. Eight of 15 patients whose open-window cavity could not be closed following an OWT procedure died in the hospital. After 2013, in addition to the above-mentioned procedures, endoscopic fistula closure was performed in 2, negative pressure wound therapy (NPWT) in 2, and concomitant use of both procedures in 3 cases. One case of endoscopic closure avoided OWT because of bronchial occlusion. Among 6 patients who underwent OWT, 5 treated with negative pressure wound therapy were cured and discharged from the hospital.

Conclusions: A variety of surgery procedures were performed during the study period for treating empyema with fistula. However, a cure could not be achieved in a significant number of those patients. We consider that multimodal approaches including endoscopic fistula closure and NPWT should be considered for use as new treatment strategies in such cases.

Keywords: Empyema with fistula; open-window thoracotomy; Endobronchial Watanabe Spigot (EWS); negative pressure wound therapy (NPWT)

Received: 10 November 2019; Accepted: 21 November 2019; Published: 05 October 2020.

doi: 10.21037 /jovs.2019.11.13

View this article at: http://dx.doi.org/10.21037/jovs.2019.11.13

\section{Introduction}

Empyema is a pathological state in which the thoracic cavity retains pus. Particularly, it is difficult to treat cases of pleural empyema with a fistula with communication between the empyema cavity and airway or esophagus because of the complicated clinical state. For treatment, an open-window thoracotomy (OWT), muscle flap transposition, and thoracoplasty are often performed, though the prognosis has been shown to be poor (1). Moreover, patients who are discharged with an open window cavity show markedly degraded quality of life. Recently, it was reported that an 


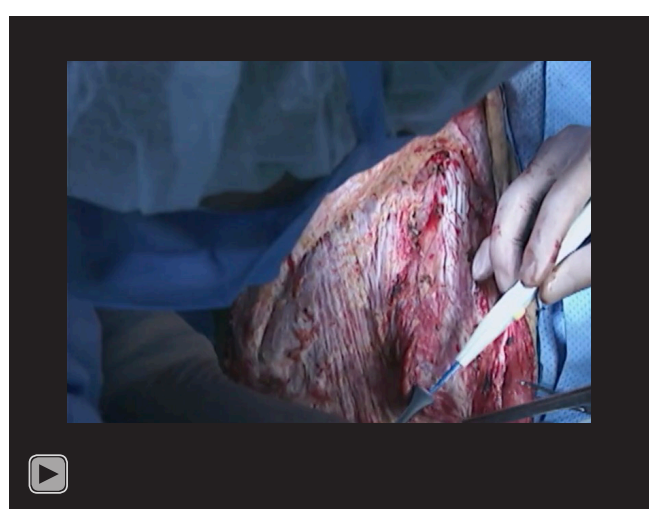

Video 1 A 58 years old man of left tuberculous empyema with fistulas underwent open-wound thoracotomy six months ago. First, latissimus dorsal muscle was meticulously dissected for a flap. Next, pleural cavity was derided and short length of both lower ribs' ends were removed for thoracoplasty. After omental flap was prepared, fistulas were closed by suturing with omental flap. Moreover latissimus dorsi muscle flap was tied over and the wound was closed without residual empyema space.

endoscopic bronchial closure procedure is effective for treating empyema with a fistula (2). Once fistula closure is attained, therapeutic intervention for treating empyema without a fistula can proceed. In another report published in 2005, negative pressure wound therapy (NPWT) was shown to be useful for open wound closure (3). In cases of empyema with fistula, following an OWT procedure, it is considered likely that window closure can be facilitated by combined use of endoscopic fistula closure and NPWT. In the present study, we retrospectively investigated surgical procedures for patients affected by empyema with a fistula who received treatment at our department. In addition, possible future treatment strategies are discussed.

\section{Methods}

From January 2003 to December 2019, 49 patients affected by empyema with a fistula underwent surgical treatment at our institution. The causative disease, surgical procedure, and prognostic data for those cases were obtained from the medical records and analyzed in a retrospective manner. The study was conducted in accordance with the Declaration of Helsinki (as revised in 2013). This study was approved by the Institutional Review Board of TNH2019032 at the Osaka Toneyama Medical Center. Individual consent for this retrospective analysis was waived due to the retrospective nature of the study.

Cases confirmed to have air in the empyema cavity by computed tomography (CT) or chest X-ray findings were considered to have empyema with a fistula. For the initial treatment, tube drainage of the empyema cavity and antibacterial administration were done for prevention of aspiration pneumonia and infection control. When drainage was insufficient, an OWT procedure was performed. Following successful OWT, radical surgery was performed, such as muscle flap transposition, thoracoplasty, omentopexy, or extra-pleural pneumonectomy, with the extent of the empyema cavity and individual patient ability to endure surgery taken into account taken into account (Video 1).

Since 2013 , in addition to the above-mentioned procedures, endoscopic fistula closure and NPWT have been utilized. The indication for bronchoscopy fistula closure is when the fistula is localized, as shown by thinslice CT. For the packing material, a polyglycolic acid sheet is rolled up tightly in accordance with the thickness of the bronchus. A silicon Endobronchial Watanabe Spigot (EWS) has been used since being listed as an acceptable filler in the Japan National Health Insurance Drug Price Standard (4). For endoscopic occlusion of an esophagopleural fistula, a clip (Olympus Corporation) for digestive endoscopy is utilized. NPWT was indicated when active infection was controlled by elimination of calcification and poor granulation in the fenestration cavity, and the fistula was closed. The equipment used was a VAC ${ }^{\circledR}$ (Kinetic Concepts, Inc.), with negative pressure starting $-75 \mathrm{mmHg}$ and gradually intensified to a maximum $-150 \mathrm{mmHg}$. The filling was replaced twice a week and treatment continued for 4 weeks.

\section{Results}

Forty-nine patients (47 males, 2 females) aged 19 to 87 years (mean 66.7 years) with empyema and a fistula were treated surgically from January 2003 to December 2018 (Table 1). The causative diseases were acid-fast bacteriumrelated infection in 19 cases (lung tuberculosis in 6, empyema associated with late effects of tuberculosis in 11 , non-tuberculosis mycobacterium infection in 2), empyema caused by pleural rupture resulting from lung aspergillosis in 8 cases, all of which showed a postpneumonectomy bronchial stump fistula and had lung cancer, esophagopleural fistula in 2 cases, lung cancer following a left pneumonectomy in 1 case, and development after a right pneumonectomy for pleural suppuration in 
Table 1 Demographic data

\begin{tabular}{lc}
\hline Variables & No. or mean \\
\hline Sex & 47 \\
Male & 2 \\
Female & $66.7(19-87)$ \\
Age, years (range) & \\
Etiology & 19 \\
Mycobacterium & 6 \\
Tuberculosis & 11 \\
Old tuberculosis & 2 \\
NTM & 8 \\
Aspergills & 8 \\
BPF & 2 \\
EPF & 12 \\
Others &
\end{tabular}

NTM, non-tuberculosis mycobacterium; BPF, broncopleural fistula; EPF, esophagopleural fistula.

Table 2 Surgical procedures

\begin{tabular}{lc}
\hline Procedures & No. \\
\hline Debridement or decortication & 55 \\
Open-window thoracotomy & 40 \\
Muscle flap & 36 \\
Thoracoplasty & 20 \\
Omentopexy & 7 \\
Pneumonectomy & 3 \\
Total & 161 \\
\hline
\end{tabular}

1 case. The other 12 were secondary cases of bacterial pneumonitis complicated with an intrapleural bacterial infection secondary to pneumothorax. Multiple courses of a variety of surgical procedures were repeated for these patients, indicating the intractable nature of the disease. A total of 161 operations were performed including cases with overlapping, including 37 open-window thoracotomy (OWT), 28 muscle flap transposition, 16 thoracoplasty, 7 omentopexy, and 3 extra-pleural pneumonectomy procedures (Table 2). The therapeutic outcomes were cure and discharge in $32(65.3 \%)$, discharge with OWT in $6(12.2 \%)$, discharge with a chest tube in $1(2.0 \%)$, and death in hospital in 10
Table 3 Total outcome

\begin{tabular}{lc}
\hline Outcome & No. (\%) \\
\hline Cure & $32(65.3)$ \\
Discharge with OWT & $6(12.2)$ \\
Discharge with a chest tube & $1(2.0)$ \\
Death in hospital & $10^{*}(20.4)$ \\
\hline
\end{tabular}

OWT, open-window thoracotomy. *, 8 of 10 cases were with OWT.

(20.4\%) cases (Table 3). Causes for in-hospital death were respiratory failure due to pneumonia exacerbation in 6 , interstitial pneumonia exacerbation in 3 , and death from a malignant lymphoma tumor in 1 . In 8 of the 10 in-hospital death cases, the open-window cavity prepared for infection control could not be closed after OWT.

Fistula closure with bronchoscopy was performed using a polyglycolic acid sheet or EWS in 4 cases (Figure 1). The first case was empyema with a fistula caused by pleural rupture due to aspergillosis, in which the fistula was occluded by muscle flap transposition followed by endoscopic placement of a polyglycolic acid sheet to form a bale-shaped filler into the responsible bronchus, which resulted in cure without OWT. The remaining 3 cases underwent OWT, 2 of which were cured. Although muscle flap transposition and thoracoplasty procedures were performed after an omentopexy in 1 of those 2 cases, fistula closure could not be achieved. Accordingly, fistula occlusion and reduction of the open-window cavity were attempted using concomitant endoscopic EWS packing and NPWT, then muscle flap transposition was added again and cure was attained. The other case was treated with muscle flap transposition and thoracoplasty procedures after OWT, though fistula closure was not attained. However, concomitant endoscopic EWS and NPWT, as well additional omentopexy, muscle flap transposition, and thoracoplasty procedures resulted in a cure for this patient. As for the case without cure, occlusion with a polyglycolic acid sheet applied with bronchoscopy was attempted though unsuccessful, because the fistula size was larger than $10 \mathrm{~mm}$ at 2 sites, the right inferior lobe bronchus stump and middle lobe bronchus, for which OWT was repeated, resulting in discharge without complete healing. For the case with an esophagopleural fistula that developed soon after performance of a right pneumonectomy, omentopexy, and muscle flap transposition following OWT, complete fistula closure could not be achieved. Accordingly, an endoscopic fistula closure procedure was performed with use of a 


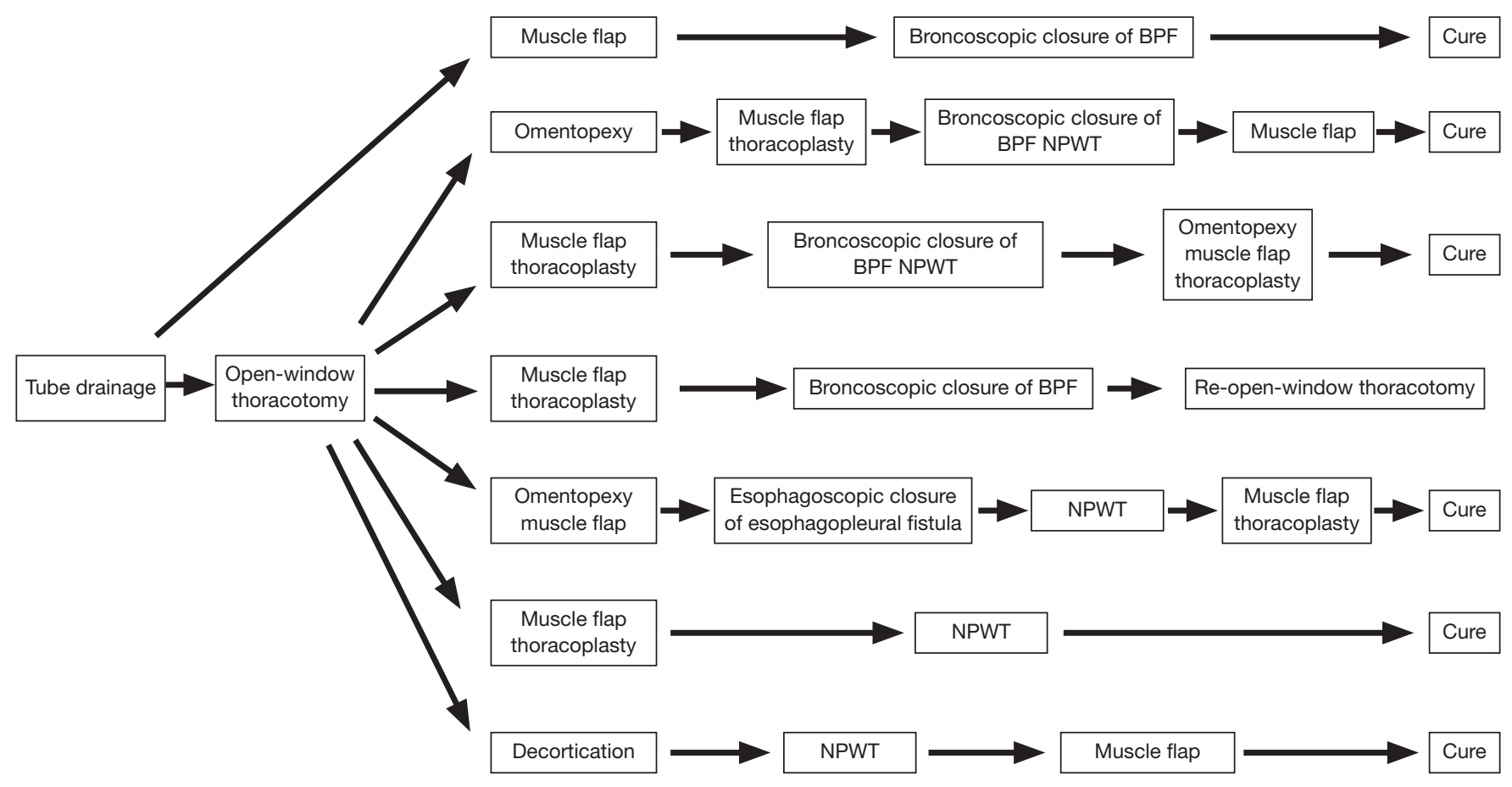

Figure 1 Clinical course of patients treated with bronchoscopic or esophagoscopic closure of fistulas or/and NPWT. Six of seven patients were cured after the treatments.

hemostatic clip for esophagoscopy, which was successful. Eventually, the empyema cavity was reduced by NPWT to close the OWT space and cure was attained.

NPWT was performed in 5 cases. In 3 of those, the above-mentioned bronchoscopic and esophagoscopy fistula closure procedures were performed in combination, while NPWT was done after natural fistula closure in the other 2, which resulted in cure and discharge from the hospital.

\section{Discussion}

Empyema with a fistula is a clinical state associated with overwhelming pulmonary, bronchial, and esophageal fistula development in the empyema cavity. It is difficult to control infection in affected patients with only empyema cavity drainage and antimicrobial administration, thus OWT is frequently employed. For radical treatment after attaining infection control, fistula occlusion is essential. In the consensus guidelines for management of empyema provided by the American Association for Thoracic Surgery, it is recommended to close as much as possible when a bronchopleural fistula is present (5). Conventionally, for closing the fistula, muscle flap transposition including the latissimus dorsi muscle and omentopexy are generally performed (1). In the present study, we analyzed a total of 161 surgical procedures performed for 49 patients (Table 2), with a cure rate of only $65.3 \%$, which was not considered to be satisfactory (Table 3). Notably, among 15 patients for whom OWT was performed early for infection control, closure was eventually achieved, though 8 died while in the hospital. Although OWT is a highly effective procedure, it has a large negative impact on patient QOL. Furthermore, once fenestration is made, the period until closure is prolonged and closure can become difficult depending on the clinical state of the patient. Thus, great challenges remain $(6,7)$.

For avoiding an OWT, we performed endobronchial fistula occlusion in a case of empyema in 2013, in which the fistula was caused by an aspergillus pleural rupture. Since the pleural empyema could not be sufficiently controlled by muscle flap transposition, a polyglycolic acid sheet was formed into a small bale-shaped filler and packed into the responsible bronchus under bronchoscopy, thus avoiding OWT and resulting in cure (Figure 1). In 2015, Cardillo et al. reported bronchoscopic fistula occlusion procedures performed in 35 cases with a post-pneumonectomy bronchopulmonary fistula and a cure rate of $80 \%$. They reported that the procedure was worth attempting for 


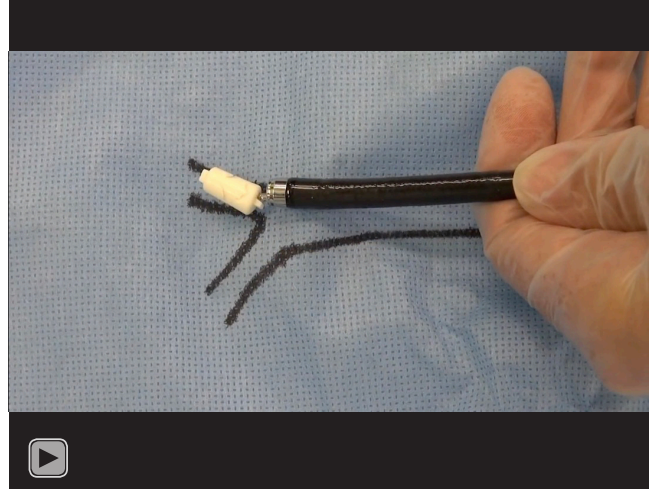

Video 2 An EWS is able to be carried to the aimed bronchus easily by attached to a tip of a curettage.

fistulas smaller than $8 \mathrm{~mm}$ (2). Accordingly, it is considered that bronchoscopic fistula closure should be considered prior to highly invasive radical surgery for cases with a smaller fistula.

At our institution, we performed bronchoscopic fistula closure in an additional 3 patients, with closure finally achieved and cure noted in 2, who were ultimately discharged. The other patient had a fistula in the right lobe bronchus stump larger than $10 \mathrm{~mm}$. Although a polyglycolic acid sheet was packed to fit the size of the fistula, closure was difficult with bronchoscopy and we consider that such a bronchial fistula larger than $10 \mathrm{~mm}$ should be excluded from the procedure indications. For the bronchial filler material, we used a silicon EWS, as reported by Watanabe (4) in 2003, in all cases, except those noted with use of a polyglycolic acid sheet. The most important point for successful treatment is how well the EWS can be packed into the bronchus. For achieving success, we mount the EWS on the tip of a curette for bronchoscopic cytodiagnosis, which allows for easy adjustment of direction and angle. Thus, an EWS can be successfully used to securely plug the target bronchus (Video 2). Morikawa $e t$ al. also recently reported success with this procedure for intractable pneumothorax (8).

For esophagopleural fistula occurrence following a pneumonectomy, Massard et al. noted that 2 of 5 patients died from complications even though they performed radical surgery procedures, such as muscle flap transposition and thoracoplasty, and concluded that such aggressive treatment might have a high risk (9). We performed fistula closure using esophagoscopy even in OWT cases after a right pneumonectomy, which resulted in disappearance of the fistula. It is considered that use of a hemostatic clip for digestive endoscopy by an experienced gastroenterologist to occlude the fistula might be effective. We were able to attain a cure in challenging cases of post-pneumonectomy esophagopleural fistula, indicating that our procedure as a promising treatment option.

For closure of open wounds, Armstrong et al. reported in 2005 a randomized control trial that included diabetic patients and the usefulness of NPWT (3). In the field of respiratory surgery, Palmen et al. in 2009 reported NPWT performed in 11 patients with empyema without a fistula whose infection could be controlled by OWT, and reported that the empyema cavity was narrowed and all were cured following muscle flap transposition (10).

It is difficult to use NPWT for cases of empyema with a fistula, as it cannot continuously retain negative pressure. However, if fistula closure can be attained by EWS, it is likely to become a prominent therapeutic option. In 3 of the present patients who had empyema with a fistula after OW' who were not be cured by conventional highly-invasive procedures, such as omentopexy, muscle flap transposition, and thoracoplasty, the fistulas were successfully closed. Of those successful cases, 2 underwent bronchoscopic fistula closure and 1 esophagoscopy fistula closure, and a cure was obtained after NPWT in each. In cases in which endoscopic fistula closure is effective, NPWT is considered to be a reasonable treatment choice. We also experienced a case in which OWT could be avoided by performing endoscopic bronchial occlusion. Although our cases are few in number, the results obtained suggest that endoscopic fistula closure and NPWT might be useful as part of a treatment strategy for empyema with a fistula.

Important limitations of the present study include its retrospective nature and the small number of subjects from a single institution. However, use of a control group in such a study is not realistic and it is difficult to enroll a large number of subjects, as cases of empyema with a fistula have a low rate of incidence, as well as various factors in regard to etiology and pathology.

\section{Acknowledgments}

Funding: None.

\section{Footnote}

Provenance and Peer Review: This article was commissioned by the editorial office, Fournal of Visualized Surgery for the series "Dedicated to the 36th Annual Conference of 
Japanese Association for Chest Surgery (JACS)". This article has undergone external peer review.

Conflicts of Interest: All authors have completed the ICMJE uniform disclosure form (available at https://jovs. amegroups.com/article/view/10.21037/jovs.2019.11.13/ coif). The series "Dedicated to the 36th Annual Conference of Japanese Association for Chest Surgery (JACS)" was commissioned by the editorial office without any funding or sponsorship. MO served as the unpaid Guest Editor of the series and serves as an unpaid editorial board member of Fournal of Visualized Surgery from August 2018 to July 2020. The authors have no other conflicts of interest to declare.

Ethical Statement: The authors are accountable for all aspects of the work in ensuring that questions related to the accuracy or integrity of any part of the work are appropriately investigated and resolved. The study was conducted in accordance with the Declaration of Helsinki (as revised in 2013). This study was approved by the Institutional Review Board of TNH-2019032 at the Osaka Toneyama Medical Center. Individual consent for this retrospective analysis was waived due to the retrospective nature of the study.

Open Access Statement: This is an Open Access article distributed in accordance with the Creative Commons Attribution-NonCommercial-NoDerivs 4.0 International License (CC BY-NC-ND 4.0), which permits the noncommercial replication and distribution of the article with the strict proviso that no changes or edits are made and the original work is properly cited (including links to both the formal publication through the relevant DOI and the license). See: https://creativecommons.org/licenses/by-nc-nd/4.0/.

\section{References}

1. Regnard JF, Alifano M, Puyo P, et al. Open window

doi: 10.21037/jovs.2019.11.13

Cite this article as: Takeuchi Y, Hayashi A, Kagawa Y, Kogita Y, Okumura M. Treatment strategy for empyema with fistulas. J Vis Surg 2020;6:39. thoracostomy followed by intrathoracic flap transposition in the treatment of empyema complicating pulmonary resection. J Thorac Cardiovasc Surg 2000;120:270-5.

2. Cardillo G, Carbone L, Carleo F, et al. The Rationale for Treatment of Postresectional Bronchopleural Fistula: Analysis of 52 Patients. Ann Thorac Surg 2015;100:251-7.

3. Armstrong DG, Lavery LA. Diabetic Foot Study Consortium. Negative pressure wound therapy after partial diabetic foot amputation: a multicenter, randomized controlled trial. Lancet 2005;366:1704-10.

4. Watanabe Y, Matsuo K, Tamaoki A, et al. Bronchial occlusion with Endobronchial Watanabe Spigot. J Bronchol 2003;10:264-7.

5. Shen KR, Bribriesco A, Crabtree T, et al. The American Association for Thoracic Surgery consensus guidelines for the management of empyema. J Thorac Cardiovasc Surg 2017;153:e129-46.

6. Hato T, Suzuki S, Harada M, et al. Comprehensive treatment approach is necessary for the closure of open window thoracotomy: an institutional review of 35 cases. Surg Today 2014;44:443-8.

7. Massera F, Robustellini M, Pona CD, et al. Predictors of successful closure of open window thoracostomy for postpneumonectomy empyema. Ann Thorac Surg 2006;82:288-92.

8. Morikawa S, Okamura T, Minezawa T, et al. A simple method of bronchial occlusion with silicone spigots (Endobronchial Watanabe Spigot; EWS®) using a curette. Ther Adv Respir Dis 2016;10:518-24.

9. Massard G, Ducrocq X, Hentz J, et al. Esophagopleural fistula: An early and long-term complication after pneumonectomy. Ann Thorac Surg 1994;58:1437-40.

10. Palmen M, van Breugel HN, Geskes GG, et al. Open Window Thoracostomy Treatment of Empyema Is Accelerated by Vacuum-Assisted Closure. Ann Thorac Surg 2009;88:1131-6. 